\title{
FATORES PREDITIVOS DE COLELITÍASE EM OBESOS MÓRBIDOS APÓS ASTROPLASTIA EM Y DE ROUX
}

\author{
Mohamed Ibrahin Ali Taha*, Wilson Rodrigues freitas Jr, Carlos Roberto Puglia, Arnaldo lacombe, Carlos Alberto Malheiros
}

Trabalho realizado na Santa Casa de Misericórdia de São Paulo, SP

\author{
*Correspondência: \\ R. Dr. José de Andrade \\ Figueira, 381, apto 132, \\ Cep: 05709-010 \\ Tel: (11) 9177-6439 \\ Fax: 5872-4722 \\ mohamedtaha@uol.com.br
}

\begin{abstract}
RESUMO
OBjetivo. Avaliar fatores preditivos de colelitíase em obesos mórbidos submetidos a gastroplastia com reconstrução em Y de Roux.

Métodos. Estudou-se um grupo de pacientes obesos inscritos no programa para tratamento cirúrgico da obesidade mórbida do Departamento de Cirurgia da Faculdade de Ciências Médicas da Santa Casa de São Paulo. Os critérios de exclusão foram: pacientes colecistectomizados previamente, pacientes com diagnóstico de colelitíase no pré-operatório e casos em que a ultra-sonografia era duvidosa em relação à presença de cálculos biliares. Foram operados 160 pacientes, sendo 29 com colecistectomia prévia, 23 com litíase biliar pré-peratória, 5 com ultrassonografia duvidosa e 103 com vesícula biliar normal ao ultra-som de abdome.
\end{abstract}

Resultados. Os resultados mostraram que 48 (46,6\%) pacientes desenvolveram colelitíase, sendo 22 sintomáticos. Quando comparamos os pacientes com e sem colelitíase, não observamos diferenças significativas em relação à idade, sexo e peso pré-operatório. O índice de massa corpórea, os níveis séricos de triglicérides, o colesterol total e suas frações VLDLcolesterol e LDL-colesterol foram superiores no grupo que desenvolveu colelitíase em relação aos pacientes sem cálculos, sendo esta diferença estatisticamente significativa. A porcentagem de perda de peso no $6^{\circ}$ e $12^{\circ}$ mês pós-operatório foi significativamente superior nos pacientes que desenvolveram cálculos biliares.

Conclusão. O estudo permite concluir que índice de massa corpórea, os níveis de triglicérides, colesterol total e suas frações LDL e VLDL são fatores preditivos de colelitíase após gastroplastia com reconstrução em Y de Roux.

Unitermos: Obesidade. Colelitíase. Gastroplastia. Obesidade mórbida. Cirurgia bariátrica.

\section{INTRODUÇÃo}

A doença biliar é comum nos obesos mórbidos, sendo a obesidade o maior fator de risco para o desenvolvimento de cálculos de colesterol ${ }^{28}$. Cerca de $25 \%$ a $45 \%$ desta população apresenta colelitíase, sendo realizadas cerca de 750.000 colecistectomias por ano nos Estados Unidos, acarretando um custo de 8 a 10 bilhões de dólares. Quando somadas as colecistectomias prévias e a colelitíase diagnosticada no pré-operatório de obesos submetidos a cirurgia bariátrica esse valor varia de $28 \%$ a $45 \% \%^{1-5}$.

A alta incidência de doença da vesícula biliar em obesos foi demonstrada por diversos autores, merecendo destaque Dittrick et $a l .^{5}$, que compararam doenças da vesícula biliar de obesos submetidos a cirurgia bariátrica e colelistectomia profilática em relação à vesícula biliar de doadores de órgãos. Seus resultados mostraram uma incidência de doença biliar em $79 \%$ dos obesos e em apenas $28 \%$ do grupo controle ${ }^{5}$.

Na Itália, um estudo epidemiológico revelou uma incidência de 17\% de cálculos assintomáticos em 4.75 I indivíduos submetidos à ultra-sonografia 6 . No Chile, que apresenta a segunda maior prevalência no mundo, estudos realizados após necropsias mostraram que mais de $50 \%$ das mulheres adultas tinham colelitíase ${ }^{7}$.
Foi evidenciada uma maior incidência de colelitíase em mulheres com IMC maior do que $30 \mathrm{~kg} / \mathrm{m}^{2}$ do que em mulheres com IMC menor do que $25 \mathrm{~kg} / \mathrm{m}^{2}$. Quando este índice foi superior a $45 \mathrm{~kg} / \mathrm{m}^{2}$, o risco de desenvolver colelitíase foi sete vezes maior ${ }^{8}$.

Outras doenças da vesícula biliar, como a colesterolose e a colecistite crônica acalculosa acometem a grande maioria dos obesos. Cirurgiões que realizaram colecistectomia de rotina, mesmo na ausência de cálculos, mostraram incidências de doença da vesícula biliar entre $79 \%$ e $95 \%$ \% 4.

Paradoxalmente, indivíduos que sofreram redução rápida de peso também apresentaram maiores riscos para desenvolver colelitíase. Os primeiros estudos que comprovaram esta teoria utilizaram dietas com poucas calorias (500 a $800 \mathrm{kgcal} / \mathrm{d}$ ), mostrando índices de $12 \%$ de colecistopatia calculosa em 16 semanas de acompanhamento 8,10 .

Com o advento da cirurgia bariátrica, e com isso a rápida perda de peso, houve um aumento na incidência de colelitíase no pós-operatório 5,6,11!

Em 1983, Wattchow foi o primeiro a relacionar um aumento da probabilidade de colelitíase após gastroplastias em Y de Roux"'. Posteriormente, vários estudos comprovaram esta relação, sendo os principais: Amaral e Thompson ${ }^{12}$ com $28 \%$ de cálculos biliares em três anos de pós-operatório, Schmidt et al..$^{13} \mathrm{com} 40 \%$, Shiffman et al. ${ }^{14} \mathrm{com}$ $47 \%$ em dois anos, Surgeman ${ }^{15}$ com $32 \%$ em seis meses e Wudel et al. ${ }^{16} \mathrm{com} 71 \%$ em um ano. 
Pela diversidade de condutas, questões foram enviadas para 123 cirurgiões da Sociedade Americana de Cirurgia Bariátrica em relação ao tipo de cirurgia bariátrica que era realizada e a opção ou não pela colecistectomia em vesículas biliares sem cálculos. Os resultados mostraram que $7 \%$ dos cirurgiões que realizavam procedimentos restritivos optavam pela colecistectomia de rotina, $30 \%$ dos que realizavam a gastroplastia em Y de Roux também faziam a colecistectomia profilática e 100\% dos que realizavam derivações biliopancreáticas removiam a vesícula ${ }^{17}$.

A relação entre colelitíase, obesidade e perda de peso está muito bem estabelecida em um grande número de estudos, porém são poucos os trabalhos que procuram determinar os fatores preditivos diretamente relacionados ao desenvolvimento de cálculos de colesterol em pacientes submetidos à perda de peso, principalmente em relação aos exames laboratoriais como colesterol e triglicérides.

Os autores tiveram como objetivo estabelecer fatores preditivos de colelitíase em obesos mórbidos submetidos à gastroplastia com reconstrução em Y de Roux.

\section{Métodos}

Este estudo foi aprovado pelo Comitê de Ética em Pesquisa da Santa Casa de São Paulo e seguiu as normas da Comissão de Pós-graduaçãa da Faculdade de Ciências Médicas da Santa Casa de São Paulo.

Estudou-se retrospectivamente um grupo de pacientes obesos, inscritos no programa para tratamento cirúrgico da obesidade mórbida do Departamento de Cirurgia da Faculdade de Ciências Médicas da Santa Casa de São Paulo, operados entre março de 2002 e janeiro de 2004.

Os critérios de exclusão foram: pacientes já colecistectomizados, pacientes com colelitíase diagnosticada no pré-operatório e casos em que a ultra-sonografia era duvidosa em relação à presença de cálculos biliares.

Foram operados 160 pacientes neste período, sendo 29 (|8, |\%) com colecistectomia prévia, 23 (I4,3\%) com litíase biliar pré-operatória, cinco (3, I\%) com ultra-sonografia duvidosa e 103 (64,3\%) com vesícula biliar normal do ponto de vista ultra-sonográfico.

Dos 103 pacientes selecionados para o estudo, 85 (82,5\%) eram do sexo feminino e $18(17,5 \%)$ do sexo masculino. A hipertensão arterial esteve presente em 59 (57,3\%), e 18 (17,5\%) pacientes eram diabéticos.

Os pacientes foram acompanhados por um ano, sendo que 48 (46,6\%) desenvolveram colelitíase no pós-operatório e 55 (53,4\%) não desenvolveram litíase biliar.

O preparo pré-operatório foi realizado por uma equipe multidisciplinar com avaliação psicológica, nutricional, clínica, cirúrgica e anestésica. Os pacientes participavam de reuniões mensais de esclarecimento.

Todos os pacientes foram submetidos à ultra-sonografia de abdome na Santa Casa de São Paulo, exame este realizado ou supervisionado por um assistente do Serviço de Diagnóstico por Imagem.
A operação realizada foi a gastroplastia com reconstrução em Y de Roux com colocação de anel de silicone de $6,4 \mathrm{~cm}$ ao redor da pequena câmara com alça alimentar de $100 \mathrm{~cm}$ e alça biliar de $50 \mathrm{~cm}$, ficando a pequena câmara gástrica com aproximadamente $30 \mathrm{~mm}$ de capacidade.

Os pacientes com colelitíase diagnosticada no pré-operatório eram submetidos à colecistectomia no mesmo ato operatório.

A dieta líquida, pouco calórica (aproximadamente $200 \mathrm{kcal} / \mathrm{dia}$ ) foi iniciada no primeiro dia de pós-operatório num volume de $30 \mathrm{~mm}$ a cada hora, mantida pelos 20 primeiros dias. Houve uma progressão lenta, chegando a pastosa, com uma média de sete refeições ao dia que perfaziam, aproximadamente, $650 \mathrm{kcal} / \mathrm{dia}$. Finalmente, após o segundo mês, foram introduzidos os alimentos sólidos, num total de cinco a seis refeições ao dia, que somavam $850 \mathrm{kcal} / \mathrm{dia}$.

No sexto mês pós-operatório, foram solicitados exames laboratoriais com ênfase no colesterol total e suas frações e nos triglicérides e ultra-sonografia de abdome. Após um ano de cirurgia, eram repetidos os exames laboratoriais e a ultrasonografia de abdome. Em todos os retornos ambulatoriais, os pacientes foram pesados e medidos, com cálculos do IMC e porcentagem de perda de peso.

No intuito de se estabelecer possíveis fatores preditivos, os seguintes parâmetros foram analisados nos dois grupos: peso, IMC, colesterol total e frações, perda de peso com dois, quatro e seis meses e com um ano de pós operatório.

Quarenta e oito pacientes tiveram diagnóstico de litíase vesicular após a gastroplastia e foram submetidos à colecistectomia após o preparo pré-operatório. Destes, 26 (54,2\%) eram assintomáticos e $22(45,8 \%)$ sintomáticos. Foram considerados sintomáticos os pacientes que apresentavam dor em andar superior do abdome, vômitos e intolerância alimentar.

A via de acesso utilizada foi a laparoscopia, sendo utilizada em 46 casos. Dois pacientes foram submetidos à colecistectomia aberta em outros serviços por colecistite aguda.

Para análise estatística, adotou-se o nível de significância de 5\% $(0,05)$, para a aplicação dos testes $t$ de Student, controlado pelo Teste de Levene para Igualdade de Variâncias, com o intuito de verificar possíveis diferenças entre os dois grupos estudados, e aplicação do Teste de Mann-Whitney, com o intuito de verificar possíveis diferenças nas distribuições das variáveis não paramétricas.

\section{Resultados}

Analisando os resultados, não observamos diferenças significativas entre os pacientes com ou sem colelitíase em relação ao sexo, idade, e peso pré-operatório. Porém, o índice de massa corpórea préoperatório foi superior nos pacientes que desenvolveram cálculos biliares, sendo esta diferença estatisticamente significativa (Tabela I).

Os níveis séricos de triglicérides e o colesterol total e suas frações $\mathrm{LDL}$ e VLDL-colesterol foram superiores no grupo que desenvolveu colelitíase com diferença estatisticamente significativa (Tabela 2).

Quando analisamos a porcentagem de perda de peso, observamos que no $6^{\circ}$ e no $12^{\circ}$ mês de pós-operatório a perda de peso foi significativamente superior no grupo com colelitíase (Tabela 3). 


\begin{tabular}{|c|c|c|c|}
\hline Variável & $\begin{array}{l}\text { Sem colelitíase } \\
\qquad N=55\end{array}$ & $\begin{array}{c}\text { Com colelitíase } \\
\qquad N=48\end{array}$ & $\begin{array}{c}\text { Significância } \\
\text { (p) }\end{array}$ \\
\hline Peso inicial (Kg) & $124,87 \pm 23,97$ & $133,40 \pm 26,02$ & $p=0,087$ \\
\hline $\operatorname{IMC}\left(\mathrm{Kg} / \mathrm{m}^{2}\right)$ & $46,73 \pm 5,93$ & $50,64 \pm 8,33$ & $p<0,05$ \\
\hline
\end{tabular}

Fonte: Departamento de Cirurgia da Santa Casa de São Paulo, 2005

\begin{tabular}{|c|c|c|c|}
\hline \multirow[b]{2}{*}{ Variável } & $\begin{array}{l}\text { nparação entre os } \\
\text { HDL-colesterol, L }\end{array}$ & tórios do colester & \\
\hline & $\begin{array}{c}\text { Sem colelitíase } \\
\quad \mathrm{N}=55\end{array}$ & $\begin{array}{c}\text { Com colelitíase } \\
n=48\end{array}$ & $\begin{array}{c}\text { Significância } \\
(\mathrm{p})\end{array}$ \\
\hline Colesterol total (mg/dl) & $179,33 \pm 29,05$ & $226,46 \pm 35,97$ & $p<0,001$ \\
\hline HDL-colesterol (mg/dl) & $41,86 \pm 7,97$ & $41,25 \pm 7,36$ & $p=0,69$ \\
\hline LDL-colesterol(mg/dl) & || $3,9 \mid \pm 25,5$ & $154,43 \pm 32,34$ & $p<0,001$ \\
\hline Colesterol/HDL & $4,4 \mid \pm 1,03$ & $5,61 \pm 1,11$ & $p<0,001$ \\
\hline VLDL-colesterol (mg/dl) & $24,69 \pm 11,49$ & $30,57 \pm 14,18$ & $p<0,05$ \\
\hline Triglicérides (mg/dl) & $129,93 \pm 45,05$ & $176,48 \pm 71,20$ & $p<0,00$ \\
\hline
\end{tabular}

Fonte: Departamento de Cirurgia da Santa Casa de São Paulo, 2005

Tabela 3 - Diferença entre os valores médios de porcentagem de perda de peso dos pacientes sem e com colelitíase no pós-operatório

\begin{tabular}{|c|c|c|c|}
\hline Variável & $\begin{array}{c}\text { Sem colelitíase } \\
\qquad \mathbf{N}=\mathbf{5 5}\end{array}$ & $\begin{array}{c}\text { Com colelitíase } \\
n=48\end{array}$ & $\begin{array}{c}\text { Significância } \\
\text { (p) }\end{array}$ \\
\hline Perda de peso em 2 meses (\%) & $13,99 \pm 3,99$ & $14,54 \pm 3,28$ & $p=0,45$ \\
\hline Perda de peso em 4 meses (\%) & $22,4 \pm 4,31$ & $23,81 \pm 3,78$ & $p=0,08$ \\
\hline Perda de peso em 6 meses (\%) & $29,41 \pm 4,48$ & $32,14 \pm 3,88$ & $p<0,001$ \\
\hline Perda de peso em I ano (\%) & $38,14 \pm 4,66$ & $41,65 \pm 3,55$ & $p<0,001$ \\
\hline
\end{tabular}

Fonte: Departamento de Cirurgia da Santa Casa de São Paulo, 2005

\section{Discussão}

A colelitíase é comum em pacientes acima do peso, sendo a obesidade considerada o principal fator de risco para a formação de cálculos biliares. A perda de peso também está relacionada com o desenvolvimento de cálculos. Diversos trabalhos relatam a maior freqüência de colelitíase em pacientes obesos que apresentaram perda de peso em relaçãa a indivíduos de peso normal 2,6,14,18.

Estudos realizados por Amaral e Thompson ${ }^{4}$, Schmidt et al. ${ }^{12}$, Shiffman et al..$^{13}$ e Fobi et al. ${ }^{14}$ mostraram uma alta incidência de colelitíase em pacientes com obesidade mórbida submetidos à cirurgia bariátrica, variando de $28 \%$ a $35 \%$. Esses dados se assemelham aos encontrados em nosso estudo, que apresentou 33,4\% de pacientes com colelitíase no pré-operatório. O ultra-som de abdome foi realizado de rotina, mesmo na ausência de sintomas, tanto em nosso estudo como nos trabalhos destes autores , $^{4,12,1,14}$.

Nossa incidência de colelitíase após gastroplastia com reconstrução em $Y$ de Roux foi de 46,6\%. Índices semelhantes com o mesmo procedimento cirúrgico foram encontrados por Schmidt et al. ${ }^{2}$, Shiffman et al. ${ }^{13}$ e Oliveira et al. ${ }^{14}$ com 40\%, 47\% e 52\%, respectiva- mente. No entanto, Wudel et al. ${ }^{16}$ apresentaram $71 \%$ de colelitíase após um ano de acompanhamento, porém sua amostra consistia de apenas 21 pacientes, o que poderia justificar esta alta incidência.

Nos procedimentos restritivos, como a banda gástrica e a gastroplastia vertical, a associação com colelitíase pós-operatória foi de 12,1\%, segundo Deitel e Petrov ${ }^{18}$, e $30 \%$, segundo Miller ${ }^{19}$, após dois anos, demonstrando que a incidência de cálculos biliares é maior nas cirurgias com componente disabsortivo.

Acredita-se que durante a perda de peso, as taxas de todos os lipídeos biliares diminuem. Em alguns pacientes, esse decréscimo é proporcional e a saturação de colesterol mantém-se inalterada. Contudo, em outros, a diminuição da secreção de sais biliares é maior do que a do colesterol, resultando numa bile hipersaturada. Este excesso de colesterol em relação aos sais biliares e fosfolípides é atribuído à mobilização periférica do colesterol 1,20,21 .

Shiffman et al..$^{20}$ e Zapata ${ }^{7}$ comprovaram esta teoria por meio da análise da bile de pacientes submetidos à cirurgia bariátrica para perda de peso, encontrando um aumento da saturação de colesterol nos pacientes que desenvolviam colelitíase, em relação ao grupo sem cálculos biliares. 
A redução da motilidade da vesícula, avaliada indiretamente pelo seu volume, ocorre por lesões de ramos hepáticos do nervo vago durante o procedimento cirúrgico ou por uma concentração insuficiente de fosfolípides e proteínas para estimular a contração vesicular. Gebhard et al. ${ }^{22}$, Erlinger ${ }^{21}$ e Zapata et al. ${ }^{7}$ mostraram que pacientes submetidos à perda de peso apresentavam um aumento do volume vesicular com diminuiçã̃o do seu esvaziamento, sugerindo uma alteração da motilidade da vesícula biliar.

Nas cirurgias com desvio da secreção duodenal, como na reconstrução de $Y$ de Roux, ocorre uma diminuição na liberação de colecistoquinina pós-prandial com conseqüente redução da motilidade vesicular e estase biliar2!.

Quando comparamos os pacientes que desenvolveram colelitíase com aqueles que não apresentaram cálculos biliares, após um ano de gastroplastia com reconstrução de $Y$ de Roux, não foram observadas diferenças estatisticamente significativas em relação a idade, sexo e peso inicial. Estes resultados são semelhantes aos encontrados por Shiffman et al., Miller et al. e Oliveira et al.,2,18,21.

O índice de massa corpórea (IMC) que antecedeu a cirurgia foi significativamente superior nos pacientes que desenvolveram colelitíase em relação ao grupo sem cálculos, sendo este achado discordante de Oliveira et al. e semelhante aos relatos de Everhart 2,8 .

Oliveira et al. mostraram não haver relação entre o IMC e o desenvolvimento de cálculos, porém sua amostra apresentava um IMC médio de $54 \mathrm{Kg} / \mathrm{m}^{2}$, superior à nossa casuística e portanto de difícil comparação $0^{2}$. Everhart relata uma relação direta entre elevados IMC e colelitíase, justificando este achado pela maior perda de peso a que estes pacientes são submetidos após a cirurgia bariátrica8 ${ }^{8}$.

No estudo apresentado, os níveis de colesterol total foram significativamente superiores nos pacientes com colelitíase em relação aos que não desenvolveram cálculos. A fração HDL (lipoproteína de alta densidade) do colesterol não apresentou diferença estatisticamente significativa entre os pacientes estudados. No entanto, a relação colesterol total e HDL-colesterol, fator utilizado para determinar riscos de doenças cardiovasculares, foi significativamente superior nos com colelitíase. Acreditamos que este índice também possa estar relacionado com formação de cálculos. As frações LDL (lipoproteína de baixa densidade) colesterol e VLDL (lipoproteína de muito baixa densidade) colesterol apresentaram níveis mais elevados nos pacientes que desenvolveram colelitíase. Isto pode ser justificado, pois são estas as frações responsáveis pela concentração do colesterol na bile.

A hipertrigliceridemia, segundo Everhart ${ }^{8}$ e Erlinger $^{21}$, está relacionada à formação de cálculos durante a perda de peso. Nossos resultados são semelhantes aos destes autores, pois pacientes com colelitíase apresentaram níveis séricos de triglicérides superiores aos que não desenvolveram cálculos.

A proporção de perda de peso e o desenvolvimento de colelitíase foi estudada por Everhart ${ }^{8}$, a fim de determinar se porcentagens de perda de peso teriam relação com a formação de cálculos. Para isso, 237 obesos receberam dietas hipocalóricas e realizaram ultrasonografia após 16 semanas. Os resultados mostraram que perdas de peso inferiores a $24 \%$ do peso inicial tinham incidência de $8 \%$ de colelitíase, porém, quando a porcentagem de perda de peso era superior a 24\%, a calculose biliar surgiria em 20,9\% dos casos.
Estes resultados poderiam justificar as diferenças de incidências de colelitíase notadas nos diversos métodos para perda de peso, já que dietas hipocalóricas e procedimentos restritivos acarretaram menores perdas de peso quando comparados às cirurgias mistas.

Em nossa casuística, a porcentagem de perda de peso foi maior nos pacientes com colelitíase em relação aos pacientes sem cálculos, sendo esta diferença significativa no sexto mês (32, $14 \%$ versus $29,41 \%$ ) e no primeiro ano $(41,65 \%$ versus $38,14 \%)$ de acompanhamento. Nossos resultados são semelhantes aos de Wudel et al. ${ }^{16}$, que realizaram o mesmo procedimento cirúrgico, encontrando uma maior perda de peso em pacientes que apresentaram colelitíase. No entanto, Shiffman et al. ${ }^{14}$ não encontraram diferenças de perda de peso absoluto ou porcentagem de perda de peso nos pacientes submetidos à gastroplastia com reconstrução em Y de Roux, que desenvolveram ou não cálculos biliares.

Quando analisamos o período de formação de cálculos, observamos que $66,7 \%$ dos casos foram diagnosticados no sexto mês após a cirurgia e apenas 33,3\% após um ano. Resultados semelhantes foram encontrados na literatura, e podem ser justificados pela maior perda de peso e concentração de colesterol na bile nos primeiros meses pós-operatórios.

Deitel e Petrov ${ }^{19}$ relataram I 4\% de sintomas biliares após dois anos de acompanhamento de pacientes submetidos à cirurgia bariátrica. Shiffman et al. ${ }^{20}$ e Wudel et al. ${ }^{16}$ apresentaram $41 \%$ de cálculos sintomáticos após gastroplastia com reconstrução em Y de Roux.

Em nosso estudo, 45,8\% dos pacientes que apresentavam colelitíase eram sintomáticos, resultados semelhantes aos de Shiffman et al. ${ }^{20}$ e Wudel et al. ${ }^{16}$ Estes dados mostram que a incidência de sintomas biliares após gastroplastia com reconstrução em Y de Roux é maior do que em indivíduos de peso normal com litíase biliar.

Apesar das altas taxas de cálculos biliares após a redução de peso por meio da cirurgia bariátrica, condutas divergentes são realizadas pelos cirurgiões. Os defensores da colecistectomia profilática argumentam que, além da alta incidência de colelitíase, este procedimento é factível com baixa morbidade e mínimo aumento no tempo cirúrgico. Fobi et al. ${ }^{4}$ relataram um aumento de 15 minutos no tempo cirúrgico com a colecistectomia, não apresentando complicações relacionadas a este procedimento em mais de 1.000 casos operados. A dificuldades técnicas de diagnóstico da colelitíase em obesos mórbidos, bem como a alta relação de alterações histológicas da vesícula biliar de obesos, relatadas por Csendes et al. e Fobi et al., seriam minimizadas com a colecistectomia profilática ${ }^{4,9}$.

Os defensores da não realização da colecistectomia relatam uma dificuldade operatória na sua realização em pacientes com IMC elevado. Hamad et al. ${ }^{23}$ observaram um aumento significativo no tempo cirúrgico e período de internação nos pacientes submetidos à colecistectomia profilática associada à gastroplastia com reconstrução em Y de Roux. A possibilidade de desaparecimento de cálculos de colesterol após a estabilização de peso e a baixa população de pacientes sintomáticos são outros fatores contra a colecistectomia de rotina.

A prevenção contra a formação de cálculos biliares após a redução de peso tem sido feita com o ácido ursodeoxicólico (ursacol), com bons resultados. Wudel et al. ${ }^{16}$ e Miller et al. ${ }^{18}$ mostraram uma redução significativa na formação de cálculos com o uso desta substância. 
TAHA MIA ET AL.

\section{Conclusão}

O ideal seria determinar qual a população de obesos que apresentaria maior risco de desenvolver cálculos após o emagrecimento, para que pudéssemos agir neste grupo indicando a colecistectomia profilática ou utilizando métodos alternativos para evitar a formação de colelitíase.

Os autores concluem que o índice de massa corpórea, o colesterol total e suas frações $L D L$ e VLDL e níveis séricos de triglicérides foram fatores preditivos de colelitíase após gastroplastia com reconstrução em Y de Roux, realizada em obesos mórbidos.

A maior porcentagem de perda de peso no pós-operatório esteve relacionada com a formação de cálculos biliares.

\section{Conflito de interesse: não há.}

\section{SUMMARY}

Predictive factors for cholelithiasis in morbidly obese SUBMITTED TO $Y$ DE ROUX GASTROPLASTY

OBJECTIVE. This study intended to evaluate predictive factors for cholelithiasis in morbidly obese submitted to gastroplasty with "Y" de Roux reconstruction.

METHODS. The population under study was a group of obese patients enrolled in a program of surgical procedure for morbid obesity at the Department of Surgery, College of Medical Sciences, Santa Casa de São Paulo. The exclusion criteria were: patients previously cholecystectomized, patients with diagnosis of pre-operative cholelithiasis and patients with a questionable ultrasonography about existence of billiary stones.

A total of I 60 patients were operated, 29 with priorcholecystectomy, 23 with pre-operative biliar lithiasis, 5 with questionable ultrasonography about billiary stones and 103 with normal gallbladders at abdominal ultrasonography.

RESULTS. Results showed that (46.6\%) of patients developed cholelithiasis, 22 of them symptomatic. When patients with or without cholelithiasis were compared, no significant difference related to age, genderand preoperative weight was observed.

The body mass index, the triglycerides serum levels, total cholesterol and the fractions HDL-cholesterol and LDL-cholesterol were higher in the group who developed cholelithiasis compared to patients without gallstones and this difference was statistically significant.

The percentage of weight loss in the $6^{\text {th }}$ and $12^{\text {th }}$ postoperative month was significantly higher in patients who developed billiary stones.

CONCLUSION. The studyleads to conclude that bodymassindex, levels of triglycerides, total cholesterol and the fractions $L D L$ and VLDL are predictive factors for cholelithiasis post-gastroplasty with the "Y" de Roux reconstruction. [Rev Assoc Med Bras 2006; 52(6): 430-4]

KEY wORDs: Obesity. Cholelithiasis. Gastroplasty. Morbid obesity. Bariatric surgery.

\section{REFERÊNCIAS}

I. Almeida AZ, Valente DC, Barroso FL. Colelitíase após Cirurgia Bariátrica. In: Garrido Jr, AB. Cirurgia da obesidade, cirurgia da obesidade. São Paulo: Atheneu; 2002. p.25I-4.
2. Oliveira CIB, Chaim EA, Silva BB. Impact of rapid weight reduction on risk of cholelithiasis after bariatric surgery. Obes Surg. 2003; I 3:625-8.

3. Nakeeb A, Comuzzie AG, Martin L, Sonnenberg GE, Swartz-Basile D, Kissebah AH. Gallstones genetics versus environment. Ann Surg. 2002;235:842-9.

4. Fobi M, Lee H, Igwe D, Felahy B, James E, Stanczyk M. Prophylactic cholecystectomy with gastric bypass operation: incidence of gallbladder disease. Obes Surg. 2002; I 2:350-3.

5. Dittrick GW, Thompson JS, Campos D, Bremers D, Sudan D. Gallbladder pathology in morbid obesity. Obes Surg. 2005; 1 5:238-42.

6. O'Brien PE, Dixon JB. A rational Approach to cholelithiasis in bariatric surgery. Arch Surg 2003; 138:908-12.

7. Zapata R, Severín C, Manríquez M, Valdivieso V. Gallbladder motility and lithogenesis in obese patients during diet-induced weight loss. Dig Dis Sci. 2000;45:42I-8

8. Everhart JE. Contributions of obesity and weight loss to gallstone disease. Ann Intern Med. 1993; 1 19:1029-35.

9. Csendes A, Burdiles P, Smok G, Csendes P, Burgos A, Recio M. Histologic findings of gallbladder mucosa in 87 patients with morbid obesity without gallstones compared to 87 control subjects. J Gastrointest Surg. 2003;7:547-5I.

10. Stampfer MJ, Maclure KM, Colditz GA, Manson JE. Willet WC. Risk of symptomatic gallstones in women with severe obesity. Am J Clin Nutr. 1992;55:652-8.

I I. Wattchow DA, Hall JC, Whiting MJ, Bradley B, lannos J, Watts JM. Prevalence and treatment of gallstones after gastric bypass surgery for morbid obesity. Br Med J. 1983;286:763.

12. Amaral JF, Thompson WR. Gallbladder disease in the morbidly obese. Am J Surg. 1985; | 49:55।-7.

13. Schmidt JH, Hocking MP, Rout WR, Woodward ER. The case for prophylactic cholecystectomy concomitant with gastric restriction for morbid obesity. Am Surg. 1988; 54:269-72.

14. Shiffman ML, Surgeman HJ, Kellum JH, Brewer WR, Moore EW. Gallstones in patients with morbid obesity: relationship to body weight, weight loss and gallbladder bile cholesterol solubility. Int J Obes Relat Metab Disord. 1993; 17:153-8.

15. Surgeman HJ, Brewer WH, Shiffman ML. A Multicenter, placebo controlled, randomized, double-blind, prospective trial of prophylactic ursodiol for the prevention of gallstones formation following gastricbypass-induced rapid weight loss. Am J Surg. 1995; 169:91-7.

16. Wudel LJ, Wright JK, Debelak JP, Allos TM, Shyr Y, Chapman WC. Prevention of gallstone formation in morbidly obese patients undergoing rapid weight loss: results of a randomized controlled pilot study. J Surg Res. 2002; 1 02:50-6.

17. Mason EE, Renquist KE. Gallbladder management in obesity surgery. Obes Surg. 2002; 12:222-9.

18. Miller K, Hell E, Lang B, Lengauer E. Gallstone formation prophylaxis after gastric restrictive procedures for weight loss. A randomized double-blind placebo: controlled trial. Ann Surg. 2003;238:697-02.

19. Deitel M, Petrov I. Incidence of symptomatic gallstones after bariatric operations. Surg Gynecol Obstet. 1987; 1 64:549-52.

20. Shiffman ML, Surgeman HJ, Kellum JH, BrewerWR; Moore EW. Gallstone formation after rapid weight loss: a prospective study in patients undergoing gastric bypass surgery for treatment of morbid obesity. Am J Gastroenterol. 1991;86: 1000-5.

21. Erlinger S. Gallstones in obesity and weight. Eur J Gastroenterol Hepatol. 2000; | 2: 1347-52.

22. Gebhard LR, Prigge WF, Ansel HJ. The role of gallbladder emptying in gallstone formation during diet-induced rapid weight loss. Hepatology. 1996;24:544-8.

23. Hamad GG, Ikramuddin S, Gourash WF, Schauer A. Elective cholecystectectomy during laparoscopic Roux-en-Y bypass: is it worth the wait? Obes Surg. 2003; 13:76-81. 